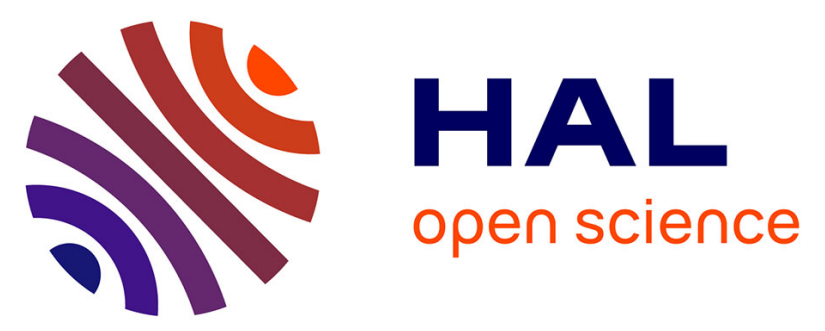

\title{
Spin-rotation and nuclear shielding constants of sulfur hexafluoride
}

\author{
Maria Laura Palma, Jose Paulo Santos
}

\section{To cite this version:}

Maria Laura Palma, Jose Paulo Santos. Spin-rotation and nuclear shielding constants of sulfur hexafluoride. Molecular Physics, 2008, 106 (09-10), pp.1241-1247. 10.1080/00268970802112145 . hal00513203

\section{HAL Id: hal-00513203 \\ https://hal.science/hal-00513203}

Submitted on 1 Sep 2010

HAL is a multi-disciplinary open access archive for the deposit and dissemination of scientific research documents, whether they are published or not. The documents may come from teaching and research institutions in France or abroad, or from public or private research centers.
L'archive ouverte pluridisciplinaire HAL, est destinée au dépôt et à la diffusion de documents scientifiques de niveau recherche, publiés ou non, émanant des établissements d'enseignement et de recherche français ou étrangers, des laboratoires publics ou privés. 


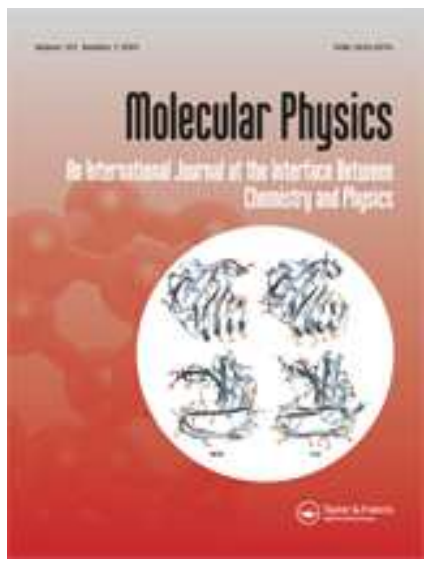

\section{Spin-rotation and nuclear shielding constants of sulfur hexafluoride}

\begin{tabular}{|c|c|}
\hline Journal: & Molecular Physics \\
\hline Manuscript ID: & TMPH-2008-0035.R2 \\
\hline Manuscript Type: & Full Paper \\
\hline $\begin{array}{r}\text { Date Submitted by the } \\
\text { Author: }\end{array}$ & 05-Apr-2008 \\
\hline Complete List of Authors: & $\begin{array}{l}\text { Palma, Maria; Faculdade de Ciências, Física } \\
\text { Santos, Jose Paulo; Universidade Nova Lisboa, Physics }\end{array}$ \\
\hline Keywords: & spin-rotation constants, hexafluoride, Ab initio calculations \\
\hline \multicolumn{2}{|c|}{$\begin{array}{l}\text { Note: The following files were submitted by the author for peer review, but cannot be converted } \\
\text { to PDF. You must view these files (e.g. movies) online. }\end{array}$} \\
\hline $\begin{array}{l}\text { SF6_Elsevier_JP_FV.tex } \\
\text { elsart-num.bst } \\
\text { jps.bib }\end{array}$ & \\
\hline
\end{tabular}

\section{今) ScholaroNE" \\ Manuscript Central}




\title{
Spin-rotation and nuclear shielding constants of sulfur hexafluoride
}

\author{
M. L. Palma ${ }^{a}$ and J. P. Santos ${ }^{b}$ \\ a Centro de Física Atómica and Dep. de Física, Faculdade de Ciências, \\ Universidade de Lisboa, Campo Grande,1749-016 Lisboa, Portugal \\ ${ }^{b}$ Centro de Física Atómica and Dep. de Física, Faculdade de Ciências e \\ Tecnologia, Universidade Nova de Lisboa, Monte de Caparica, 2825-114 Caparica, \\ Portugal \\ (Received 6 February 2008; final version received)
}

\begin{abstract}
We present a first theoretical determination of the hyperfine coupling constants of a spherical top molecule, using $a b$ initio methods. The scalar and the tensorial contributions to the spin-rotation constants and the diamagnetic and paramagnetic contributions to the nuclear shielding constant are calculated for the ${ }^{32} \mathrm{SF}_{6}$ molecule. The corrections to the spin-rotation constants due to the nuclear Thomas precession are evaluated and discussed. Our results are compared with previously reported experimental values.
\end{abstract}

Key words: Ab initio calculations, spin-rotation constants, hexafluoride PACS:

\section{Introduction}

Studies concerning the molecule $\mathrm{SF}_{6}$ are of considerable interest from several points of view. For example, control of $\mathrm{SF}_{6}$ in the atmosphere is fundamental for environmental security in such way, that the Kyoto protocol regulates the allowable values. Effectively, in spite of being non-toxic, non-corrosive, and a chemical gas that does not destroy the ozone layer, it has a powerful greenhouse effect and a global warming power, much larger than $\mathrm{CO}_{2}$ over a longtime horizon.

The $\mathrm{S}^{19} \mathrm{~F}_{6}$ molecule is an octahedral type molecule with a central spinless $\mathrm{S}$ atom and outside $1 / 2$ spin $\mathrm{F}$ nuclei. The study of magnetic hyperfine structures

Preprint submitted to Elsevier Science $\quad 5$ April 2008 
detected in highly symmetric molecules like this has progressed enormously in the seventh and eighth decades of last century. Some of the fundamental tools for that progress were the ultra-high resolution infrared spectroscopy [1-3] and the development of a tensorial formalism suitable for the interpretation of the measured spectra [4-8]. However, the theoretical determination of the electronic contribution to the nuclear hyperfine constants included in the hamiltonian that describes the magnetic hyperfine structures, could not be achieved in those days due to the impossibility of achieving sufficiently accurate calculations. Therefore, comparison with experimental results could not be completely done.

Recent progress on derivative techniques together with ab initio methods have enhanced the possibility of performing those kind of calculations. Particularly, for the spin-rotation constants, it has been shown by Gauss, Ruud and Helgaker [9] that the combination of derivative techniques with rotational London orbitals gives a suitable method of calculation.

In the present work we have used derivative techniques and $a b$ initio methods to calculate the spin-rotation constants of the $\mathrm{SF}_{6}$ molecule. We have calculated separately the nuclear and electronic contribution to these constants and discussed the influence of the Thomas precession factor as well [10]. In addition, we have calculated the shielding tensor, and discussed its relationship with the spin-rotation tensor, as firstly established by Ramsey [11] for diatomic molecules and generalized by Flygare for polyatomic molecules [12]. We have also discusses the basis sets more suitable for the study of the $\mathrm{SF}_{6}$ molecule. The utilization of rotational London orbitals has been proven to be essential to get significative results. A comparison with the available experimental results is presented here. Effects due to the molecule vibration will not be considered in this work.

This work represents the first step on the calculation of spin-rotation and shielding constants of spherical top molecules using ab initio methods and derivative techniques.

\section{Formalism and conventions}

\subsection{The spin-rotation hamiltonian}

The study of magnetic interactions in molecules in their ground electronic state, and particularly of the spin-rotation interaction and its relationship with paramagnetic shielding, has been extensively developed in early works where a fundamental physical description of these interactions has been es- 
where $\mathbf{I}_{N}$ is the nuclear spin angular momentum of nucleus $N$.

The tensor ${ }^{N} \mathbf{C}$ may be represented by the sum of a purely nuclear contribution, ${ }^{N} \mathbf{C}^{n}$, and an electronic contribution, ${ }^{N} \mathbf{C}^{e}$, depending upon the electronic variables,

$$
{ }^{N} \mathbf{C}={ }^{N} \mathbf{C}^{\mathrm{n}}+{ }^{N} \mathbf{C}^{\mathrm{e}}
$$

where, the nuclear contribution is given by

$$
{ }^{N} \mathbf{C}^{n}=\sum_{K \neq N} \frac{\hbar g_{N} Z_{K} \mu_{n} e}{4 \pi \varepsilon_{0} c^{2}}\left\{\left(\frac{\mathbf{r}_{N K}}{\mathbf{r}_{N K}^{3}} \cdot\left[\gamma_{N}^{T} \mathbf{r}_{N}-\mathbf{r}_{K}\right]\right) \mathbf{I}^{-1}-\left[\gamma_{N}^{T} \mathbf{r}_{N}-\mathbf{r}_{K}\right] \frac{\mathbf{r}_{N K}}{r_{N K}^{3}} \cdot \mathbf{I}^{-1}\right\}
$$

Here, $Z_{N}, r_{N}, m_{N}$ and $g_{N}$ are, respectively, the atomic number, position, mass and the nuclear $g$ factor of the $N$ nucleus. $e, \mu_{n}$ and $c$ are the electronic charge, nuclear magneton $\mu_{n}=\frac{e \hbar}{2 m_{p}}$ and velocity of light, respectively. $m_{p}$ is the mass of the proton, and $\mathbf{I}$ is the inertia tensor.

The electronic contribution is equal to the sum of a diamagnetic term, ${ }^{N} \mathbf{C}^{\text {ed }}$, involving only the electronic functions of the fundamental electronic state, and a paramagnetic term, ${ }^{N} \mathbf{C}^{\mathrm{ep}}$, involving those of the other electronic states,

$$
{ }^{N} \mathbf{C}^{\mathrm{e}}={ }^{N} \mathbf{C}^{\mathrm{ed}}+{ }^{N} \mathbf{C}^{\mathrm{ep}}
$$


where

$$
{ }^{N} \mathbf{C}^{\mathrm{ed}}=\sum_{i} \frac{\hbar e g_{N} \mu_{n}}{4 \pi \varepsilon_{0} c^{2}} \gamma_{N}^{T}\left\langle 0\left|\left(\frac{\mathbf{r}_{i N}}{r_{i N}^{3}} \cdot \mathbf{r}_{N}\right) \mathbf{I}^{-1}-\mathbf{r}_{N}\left(\frac{\mathbf{r}_{i N}}{r_{i N}^{3}} \cdot \mathbf{I}^{-1}\right)\right| 0\right\rangle .
$$

and

$$
{ }^{N} \mathbf{C}^{\mathrm{ep}}=\sum_{p \neq 0} \frac{\hbar e g_{N} \mu_{n}}{4 \pi \varepsilon_{0} m c^{2}}\left(V_{0}-V_{p}\right)^{-1}\left[\left\langle 0\left|\mathbf{L}^{\prime}\right| p\right\rangle\left\langle p\left|\mathbf{L} \cdot \mathbf{I}^{-1}\right| 0\right\rangle+\left\langle 0\left|\mathbf{L} \cdot \mathbf{I}^{-1}\right| p\right\rangle\left\langle p\left|\mathbf{L}^{\prime}\right| 0\right\rangle\right] .
$$

The index $i$ stands for an electron, $m$ for its mass, $|0\rangle$ and $|p\rangle$ represents the fundamental electronic state and an arbitrary electronic $p$ state, respectively.

The electronic angular momentum, L, and the closely related operator $\mathbf{L}^{\prime}$, are given, respectively, by

$$
\mathbf{L}=\sum_{i} \mathbf{r}_{i} \times \mathbf{p}_{i}
$$

and

$$
\mathbf{L}^{\prime}=\sum_{i} \frac{\mathbf{r}_{i N}}{\mathbf{r}_{i N}^{3}} \times \mathbf{p}_{i}
$$

The correction due to Thomas precession [10] corresponds to the introduction of the following corrective factor in the diamagnetic contribution to the spinrotation tensor

$$
\gamma_{N}^{\mathrm{T}}=\left(1-\frac{Z_{N} m_{\mathrm{p}}}{g_{N} m_{N}}\right)
$$

In the above expressions for the coefficients of the spin-rotation tensor all vector quantities are relative to the molecular center of mass.

It has been shown by Flygare [12] that it is possible to eliminate the term given by Equation (5) by referring the operators defined by Equations (7) and (8) to the $N$ nucleus. The aim of such tranformation, which has been extensively used in later works, is to obtain a more easily calculable relation between the spin-rotation and the shielding tensors. However, this transformation was not used in this work since the paramagnetic terms are evaluated around the center of mass.

It should be mentioned that the evaluation of expressions like Equation (6) corresponding to second-order corrections to the electronic hamiltonian operator, usually called second-order properties, is very inefficient in ab initio methods; these second-order properties are usually calculated by derivative methods based on the Hellman-Feynman theorem.

Specifically, the calculation of the second derivatives of the electronic energy 
$E^{\text {et }}$ with respect to $I$ and $J$ gives a suitable method to obtain this contribution $[9]$,

$$
{ }^{N} \mathbf{C}^{\mathrm{e}}=-\left(\frac{\partial^{2} E^{\mathrm{et}}}{\partial I_{N} \partial J}\right)_{I_{N} J=0}
$$

\subsection{Symmetry considerations}

The non-vanishing elements of the tensor ${ }^{N} \mathbf{C}$ (and obviously of each of the tensors ${ }^{N} \mathbf{C}^{\mathrm{n}}$ and ${ }^{N} \mathbf{C}^{\mathrm{e}}$ ) are determined by symmetry considerations of the magnetic field detected at each nuclear site. In the case of an octahedral-type molecule like the $\mathrm{SF}_{6}$, we can follow the discussion given in reference [5].

Let us consider a molecule fixed frame, Oxyz, with two F nuclei symmetrically located on each axis (two fluor nucleus, $\mathrm{F}_{1}$ and $\mathrm{F}_{2}$, at positions $+a,-a$ on the $x$ axis, two fluor nucleus, $\mathrm{F}_{3}$ and $\mathrm{F}_{4}$, at positions $+a,-a$ on the $y$ axis and two fluor nucleus, $F_{5}$ and $\mathrm{F}_{6}$, at positions $+a,-a$ on the $z$ axis), and the $\mathrm{S}$ nucleus at the origin.

The $J_{\alpha}(\alpha=x, y$ or $z)$ angular momentum component will generate a magnetic field directed along the $\alpha$ axis. Moreover, for a nucleus on the $\gamma$ axis (with $\gamma \neq \alpha, \beta)$, the $J_{\alpha}$ and $J_{\beta}$ components will create identical effects; $(\alpha, \beta, \gamma) \equiv$ $(x, y, z)$. It is then easily shown that the ${ }^{N} \mathbf{C}$ tensor may be written as a sum of a scalar and a tensorial contribution,

$$
{ }^{N} \mathbf{C}=c_{\mathrm{a}} \mathbf{1}+\frac{1}{3} c_{\mathrm{d}}{ }^{N} \varepsilon
$$

where $c_{\mathrm{a}}$ and $c_{\mathrm{d}}$ are, respectively, the scalar, or average spin-rotation constant, and the tensor spin-rotation constant, or anisotropy in the spin-rotation tensor. 1 is the identity tensor and ${ }^{N} \varepsilon$ are second-rank diagonal traceless tensors with elements ${ }^{N} \varepsilon_{\alpha \alpha}=-2$, if the nucleus $N$ is on axis $\alpha$, and ${ }^{N} \varepsilon_{\alpha \alpha}=1$, if the nucleus $N$ is on axis $\beta$ or $\gamma(\beta, \gamma \neq \alpha)$. More specifically, in the case of the adopted $O x y z$ frame,

$$
{ }^{1} \varepsilon={ }^{2} \varepsilon=\left(\begin{array}{ccc}
-2 & 0 & 0 \\
0 & 1 & 0 \\
0 & 0 & 1
\end{array}\right) ;{ }^{3} \varepsilon={ }^{4} \varepsilon=\left(\begin{array}{ccc}
1 & 0 & 0 \\
0 & -2 & 0 \\
0 & 0 & 1
\end{array}\right) ;{ }^{5} \varepsilon={ }^{6} \varepsilon=\left(\begin{array}{ccc}
1 & 0 & 0 \\
0 & 1 & 0 \\
0 & 0 & -2
\end{array}\right)
$$

and the $c_{\mathrm{a}}$ can be obtained from the ${ }^{N} \mathbf{C}$ tensor of any nuclei $N$,

$$
c_{\mathrm{a}}=\frac{1}{3} \operatorname{Tr}{ }^{N} \mathbf{C} .
$$


For either of the two nuclei $N$ on the axis $\alpha$ with $\beta \neq \alpha$, we have

$$
c_{\mathrm{d}}={ }^{N} C^{\beta \beta}-{ }^{N} C^{\alpha \alpha} .
$$

According to Equation (2), we have

$$
c_{\mathrm{a}}=c_{\mathrm{a}}^{\mathrm{n}}+c_{\mathrm{a}}^{\mathrm{e}}
$$

and

$$
c_{\mathrm{d}}=c_{\mathrm{d}}^{\mathrm{n}}+c_{\mathrm{d}}^{\mathrm{e}},
$$

where $c_{\mathrm{a}}^{\mathrm{n}}\left(c_{\mathrm{a}}^{\mathrm{e}}\right)$ and $c_{\mathrm{d}}^{\mathrm{n}}\left(c_{\mathrm{d}}^{\mathrm{e}}\right)$ are the scalar and the tensorial contribution to the nuclear (electronic) spin-rotation constants, respectively. For $c_{\mathrm{a}}^{\mathrm{e}}$ and $c_{\mathrm{d}}^{\mathrm{e}}$, we have also the corresponding decomposition in diamagnetic and paramagnetic parts, i.e.,

$$
c_{\mathrm{a}}^{\mathrm{e}}=c_{\mathrm{a}}^{\mathrm{ed}}+c_{\mathrm{a}}^{\mathrm{ep}}
$$

and

$$
c_{\mathrm{d}}^{\mathrm{e}}=c_{\mathrm{d}}^{\mathrm{ed}}+c_{\mathrm{d}}^{\mathrm{ep}} .
$$

\subsection{The shielding hamiltonian}

Ramsey in his seminal paper entitled "Magnetic shielding of nuclei in molecules" [11] has shown that the effect of shielding can be expressed as a sum of a diamagnetic term, similar to the Lamb expression for atoms, and a second order paramagnetic term. The evaluation of this paramagnetic term has motivated several works, many of them taking advantage of its relationship with the spin-rotation interaction measurement results.

The shielding hamiltonian may be represented by

$$
\mathbf{H}_{\mathrm{SH}}=\mu_{n} \sum_{N} g_{N} \mathbf{I}_{N} \cdot{ }^{N} \sigma \cdot \mathbf{B}
$$

where $\mathbf{B}$ is the external applied magnetic field. There is a diamagnetic, ${ }^{N} \sigma^{\mathrm{d}}$, and a paramagnetic contribution, ${ }^{N} \sigma^{\mathrm{p}}$, to the shielding tensor ${ }^{N} \sigma$,

$$
{ }^{N} \sigma={ }^{N} \sigma^{\mathrm{d}}+{ }^{N} \sigma^{\mathrm{p}}
$$

where

$$
{ }^{N} \sigma^{\mathrm{d}}=\sum_{i} \frac{e^{2}}{8 \pi \varepsilon_{0} m c^{2}}\left\langle 0\left|\frac{\mathbf{r}_{i N} \cdot \mathbf{r}_{i}}{\mathbf{r}_{i N}^{3}} \mathbf{1}-\frac{\mathbf{r}_{i N}}{\mathbf{r}_{i N}^{3}} \mathbf{r}_{i}\right| 0\right\rangle
$$

and

$$
{ }^{N} \sigma^{\mathrm{p}}=-\frac{e^{2}}{8 \pi \varepsilon_{0} m^{2} c^{2}} \sum_{p \neq 0}\left(V_{p}-V_{0}\right)^{-1}\left\{\left\langle 0\left|\mathbf{L}^{\prime}\right| p\right\rangle\langle p|\mathbf{L}| 0\rangle+\left[\left\langle 0\left|\mathbf{L}^{\prime}\right| p\right\rangle\langle p|\mathbf{L}| 0\rangle\right]^{*}\right\} .
$$


The relationship between the paramagnetic component of the shielding and the spin-rotation tensors for this kind of molecule is directly obtained by comparing Equation (6) and Equation (22),

$$
{ }^{N} \sigma^{\mathrm{p}}={\frac{1}{2 \gamma_{N}}}^{N} \mathbf{C}^{\mathrm{ep}} \cdot \mathbf{I},
$$

where $\gamma_{N}$ is given by (in S.I.) $\gamma_{N}=\frac{m}{e} \hbar \mu_{n} g_{N}$.

Therefore, we may write the following simple relationship between the spinrotation and the shielding tensors:

$$
{ }^{N} \mathbf{C}-{ }^{N} \mathbf{C}^{\mathrm{n}}={ }^{N} \mathbf{C}^{\mathrm{ed}}+2 \gamma_{N}\left({ }^{N} \sigma-{ }^{N} \sigma^{\mathrm{d}}\right) \cdot \mathbf{I}^{-1}
$$

We note that for spherical tops the inertia tensor is proportional to the identity matrix, i.e., $\mathbf{I}=I \mathbf{1}$. From symmetry considerations, the shielding tensor ${ }^{N} \sigma$ can also be written as a sum of a scalar and a tensorial part, like Equation (11). However, for performing a comparison with previous works on chemical shifts or nuclear magnetic resonance, we only present the results concerning the average diamagnetic and paramagnetic shieldings for the nucleus $\mathrm{N}$, namely

$$
{ }^{N} \sigma_{\mathrm{a}}^{\mathrm{d}}=\frac{1}{3} \operatorname{Tr}\left({ }^{N} \sigma^{\mathrm{d}}\right)
$$

and

$$
{ }^{N} \sigma_{\mathrm{a}}^{\mathrm{p}}=\frac{1}{3} \operatorname{Tr}\left({ }^{N} \sigma^{\mathrm{p}}\right)
$$

\section{Computational details}

All calculations were performed at the Hartree-Fock (HF) level on a IBM RISC/6000 workstation using the Dalton program package (Release 2.0)[15]. It was used the experimental equilibrium geometry, in which $\mathrm{SF}_{6}$ is an octahedron with central $\mathrm{S}$ nucleus, external $\mathrm{F}$ nucleus and $R_{\mathrm{S}-\mathrm{F}}=1.5605 \AA$ [16]. The nuclear $\mathrm{g}_{F}$ value was taken to be 5.26 [17].

It is important to remark that the basis set requirements for obtaining molecular coefficients concerning hyperfine interactions with a given accuracy are usually different from those required in energy calculations with the same accuracy. The values of the coefficients are not bound and so there is no analogy in the aplication of the variational principle.

The basis sets were optimized at the Hartree-Fock level. For the $\mathrm{S}$ atom the following basis were considered: the STO-3G* that includes polarization functions [18]; the DZP (Dunning), which is obtained by adding a single set of 


\section{Molecular Physics}

polarization functions ( $\mathrm{p}$-functions for hydrogen and d-functions for heavy atoms) to a Double Zeta Basis [19]; and the TZVP (DFT), which is a local spin density basis set that supports $\mathrm{S}$ and $\mathrm{F}$ atomic numbers [20].

For the $\mathrm{F}$ atom we have also used the $6-31++\mathrm{G}^{* *}$ basis sets which adds a diffuse (s,p) shell to elements from Li to Cl [21]. The DZP + Diffuse (Dunning) basis sets [19] supports also the atomic number of fluorine but not the sulfur one.

It has been proved that the use of rotational London orbitals is fundamental for obtaining a suitable basis set convergence [9].

The way the orbital connection is defined establishes the correspondence among the orbitals and the values of the perturbation strength. Molecular properties are, in principle, independent of the choice of the orbital connection adopted for the calculation of second order molecular properties. Nevertheless, that choice affects the contributions among the various terms of a given molecular property, like the diamagnetic and paramagnetic contributions to the nuclear magnetic shielding.

It has been shown [22] that only the natural connection enables the obtention of physically well defined contributions for nuclear magnetic shielding. So, we have adopted this approach in our calculations. This is an important issue since we have evaluated the correction due to Thomas precession to the electronic contribution to spin-rotation constants by using Equation (5), which correction only affects the diamagnetic contribution.

To calculate the shielding constants, we have used also the Dalton program, as well as the London atomic orbitals and the natural connection. We were restricted to the HF level since a second-order Møller-Plesset perturbation level [23] (MP2), or a density functional theory (DFT), calculation would disable the use of London orbitals.

The calculations of the spin-rotation constants, for which we have more accurate experimental results, suggest that TZVP(DFT) basis set for $\mathrm{S}$ and $\mathrm{F}$ nuclei is the more suitable basis for the calculation of magnetic properties. So, we have also used this basis to obtain the shielding constants independently of the spin-rotation constants. A discussion concerning the relation between the values obtained for both constants is presented at the end of Section 4.2.

In the case of the highly symmetric $\mathrm{SF}_{6}$ molecule $\left(O_{h}\right.$ point group), the identical six outside nuclei imposes symmetry conditions which can be adequately treated by the methods of group theory. This approach allows the direct obtention of very simple expressions relating the coefficients of the hyperfine hamiltonian. However, we could not impose a priori such high symmetry conditions in the Dalton program we have used. So, we have introduced the highest 
symmetry allowed and we have adequately treated the obtained results.

\section{Results and discussion}

\subsection{Spin-rotation constants}

A first determination of $\left|c_{a}\right|$ was obtained by Crapo and Flynn [24] from a molecular beam magnetic resonance (MBMR) nuclear moment spectrum. A more detailed study of the rotational magnetic moment spectrum of ${ }^{32} \mathrm{SF}_{6}$ by the MBMR method, reported by Ozier, Yi and Ramsey [17], produced the experimental value $c_{a}=-5.27 \pm 0.40 \mathrm{kHz}$. The sign of $c_{a}$ was determined in that work from the relationship between the spin-rotation and the nuclear shielding tensor [25].

The development of the saturation absorption spectroscopy technique has led to results with an increased precision, not only for $\mathrm{c}_{\mathrm{a}}$ but also for the tensorial spin rotation tensorial constant $c_{d}$. The more precise results obtained with this technique, to our knowledge, are $\mathrm{c}_{\mathrm{a}}=-5.140 \pm 0.010 \mathrm{kHz}[3]$ and $\mathrm{c}_{\mathrm{d}}=$ $-4.600 \pm 0.010 \mathrm{kHz}[26]$. They will be taken as standard for comparison with our theoretical results due to their high degree of accuracy.

Neglecting the correction due to the Thomas precession in Equation (3), the nuclear contribution to the scalar and tensorial spin-rotation constants are:

$$
c_{\mathrm{a}}^{\mathrm{n}}=4.314 \mathbf{k H z} \text { and } c_{\mathrm{d}}^{\mathrm{n}}=3.783 \mathbf{k H z} .
$$

The correction due to Thomas precession in the nuclear coefficients was calculated by introducing the corrective factor $\gamma_{N}^{\mathrm{T}}$ given by Equation (9). We have obtained the following results:

$$
c_{\mathrm{a}, \mathrm{T}}^{\mathrm{n}}=4.051 \mathbf{k H z} \text { and } c_{\mathrm{d}, \mathrm{T}}^{\mathrm{n}}=3.388 \mathbf{k H z}
$$

The corrections due to the Thomas precession in the electronic coefficients were calculated by multiplying the diamagnetic contributions $c_{\mathrm{a}}^{\text {ed }}$ and $c_{\mathrm{d}}^{\mathrm{ed}}$, obtained without correction from the Dalton program, by the $\gamma_{N}^{\mathrm{T}}$ factor, i. e.,

$$
c_{\mathrm{a}, \mathrm{T}}^{\mathrm{ed}}=\gamma_{N}^{\mathrm{T}} c_{\mathrm{a}}^{\mathrm{ed}} \text { and } c_{\mathrm{d}, \mathrm{T}}^{\mathrm{ed}}=\gamma_{N}^{\mathrm{T}} c_{\mathrm{d}}^{\mathrm{ed}}
$$

The electronic contributions are the sum of the diamagnetic and paramagnetic contributions. 


\section{Molecular Physics}

butions to the spin-rotation constants, using the adopted basis sets are listed in Table 1, where the subscript "T" means that the correction due to the Thomas precession was taken into account.

In Table 2, we provide the results obtained in this work for the scalar $\left(c_{\mathrm{a}, \mathrm{T}}\right)$ and tensorial $\left(c_{\mathrm{d}, \mathrm{T}}\right)$ spin-rotation constants with Thomas precession correction. Note that the sign of the electronic correction to the spin-rotation constants due to the Thomas precession is opposite to that of the corresponding nuclear correction.

In Fig. 1, we plot the relative difference, for the scalar and tensorial spinrotation constants, between the experimental values obtained by Chardonnet and Bordé $[3,26]$ and the results obtained in this work, with $\left(\Delta c_{\mathrm{a}, \mathrm{T}}, \Delta c_{\mathrm{d}, \mathrm{T}}\right)$ and without $\left(\Delta c_{\mathrm{a}}, \Delta c_{\mathrm{d}}\right)$ the Thomas precession correction, where $\Delta c_{\mathrm{x}}=\left(c_{\text {Exp }}-\right.$ $\left.c_{\mathrm{x}}\right) / c_{\operatorname{Exp}}$.

We observe that the theoretical values calculated with the TZVP basis set are the ones that most agree with the experimental results. Furthermore, among this set of values, the ones that incorporate the Thomas precession correction $\left(\Delta \mathrm{c}_{\mathrm{a}, \mathrm{T}} \simeq 15 \%, \Delta \mathrm{c}_{\mathrm{d}, \mathrm{T}} \simeq 11 \%\right)$ are more close to the experimental values, than the remaining ones $\left(\Delta c_{\mathrm{a}}=\simeq 16 \%\right.$ and $\left.\Delta \mathrm{c}_{\mathrm{d}}=15 \%\right)$. Thus, we conclude that the use of the Thomas precession correction improves the comparison between theoretical and experimental values of about $1 \%$ in the case of the scalar constant and $4 \%$ in the case of the tensorial constant; it means a correction slightly greater than $100 \mathrm{~Hz}$ for the tensorial contribution.

\subsection{Shielding constants}

The value of the diamagnetic part of the molecular shielding constant is approximately constant for a given atom from one molecule to another because the chemical environment does not affect this constant. The largest contribution is given by the inner shell electrons. For example, in what concerns the theoretical values obtained for ${ }^{19} \mathrm{~F}$, we have for the $\mathrm{F}_{2}$ molecule $\sigma^{\mathrm{d}}=529.5$ ppm, and for the HF molecule $\sigma^{\mathrm{d}}=492.4 \mathrm{ppm}$ [27]. We have obtained in this work $\sigma^{\mathrm{d}}=582.3 \mathrm{ppm}$ for the $\mathrm{SF}_{6}$ molecule.

Experimental values for the paramagnetic part of the molecular shielding constant have been early determined and discussed by Mohanty [28] using Sunney and Daas theory [29] and a generalization of the Ramsey's formula that relates the paramagnetic part of the shielding constant and the spin-rotational constant. The value obtained was $-615 \pm 26 \mathrm{ppm}$. By using the same relationship, Ozier, Yi and Ramsey [17] have evaluated the paramagnetic part of the shielding constant from their experimental determination of the spin-rotation 
constant obtained by the molecular beam resonance method. These authors obtained the value $-575 \pm 66 \mathrm{ppm}$.

Unlike the case of the $\mathrm{F}_{2}$ molecule (and other similar molecules discussed in [30]) there is no antishielding for the $\mathrm{SF}_{6}$ molecule. We have obtained the value $\sigma^{\mathrm{p}}=-416.9 \mathrm{ppm}$ for the fluorine shielding paramagnetic contribution. This value may be compared with the value for the scalar contribution to the paramagnetic spin-rotation constant obtained with the same basis set, $c_{\mathbf{a}}^{\mathbf{e p}}=-6.5207 \mathbf{K H z}$ (see Table 1). The evaluation of $\sigma^{\mathbf{p}} / c_{\mathbf{a}}^{\mathbf{e p}}=$ $63.93 \times 10^{-3} \mathrm{ppm} / \mathbf{H z}$ shows that the Equations (23) and (24) are satisfied with an accuracy of $0.3 \%$.

\section{Conclusions}

We have obtained by $a b$ initio methods the values for the scalar and tensorial spin rotation constants of the spherical type $\mathrm{SF}_{6}$ molecule. The electronic contribution to these constants had only been previously evaluated through the calculation of the difference between the experimental value and the nuclear component, which is exactly obtained from the molecular constants [26].

The basis sets constructed from density functional theory have proved to be the most suitable for these kind of calculations. The use of rotational London orbitals is also crucial to obtain a good convergence.

As discussed by Ramsey, there is a non-negligible correction due to a relativistic Thomas precession resulting from the acceleration of the nuclei. We have evaluated this correction in the framework of the rigid rotor approximation, as we are not taking into account molecular vibration in this work. It has been shown that the introduction of this correction is more significant for the tensorial contribution and improves its comparison with experimental values to a relative difference of $4 \%$.

We have also calculated the diamagnetic and the paramagnetic components of the shielding tensor and compared them with experimental results from previous works. The relationship between the paramagnetic component of the shielding tensor and the spin-rotation tensor has been discussed for spherical tops.

The extension of these calculations beyond the Hartree-Fock level and the inclusion of vibrational effects are the essential tools to improve the calculated results. 
This work was partially supported by the FCT project POCTI/0303/2003 financed by the European Community Fund FEDER.

\section{References}

[1] C. J. Bordé, M. Ouhayoun, J. Bordé, J. Mol. Spectrosc. 73 (1978) 344.

[2] J. Bordé, C. J. Bordé, C. Salomon, A. V. Lerberghe, M. Ouhayoun, C. D. Cantrell, Phys. Rev. Lett. 45 (1980) 14.

[3] C. Chardonnet, C. J. Bordé, Europhys. Lett. 9 (1989) 527.

[4] F. Michelot, B. Bobin, J. Moret-Bailly, J. Mol. Spectrosc. 76 (1979) 374.

[5] J. Bordé, C. J. Bordé, Chem. Phys. 71 (1982) 417.

[6] F. Michelot, J. Mol. Spectrosc. 106 (1984) 77.

[7] F. Michelot, J. Mol. Spectrosc. 107 (1984) 160.

[8] M. L. Palma, J. Bordé, Mol. Phys. 70 (1990) 863.

[9] J. Gauss, K. Ruud, T. Helgaker, J. Chem Phys. 105 (1996) 2804.

[10] N. F. Ramsey, Phys. Rev. 90 (1953) 232.

[11] N. F. Ramsey, Phys. Rev. 78 (1950) 699.

[12] W. H. Flygare, Chem. Rev. 74 (1974) 653.

[13] F. Michelot, Mol. Phys. 45 (1982) 949.

[14] F. Michelot, Mol. Phys. 45 (1982) 971.

[15] Dalton, a molecular electronic structure program, release 2.0 (2005), see http://www.kjemi.uio.no/software/dalton/dalton.html.

[16] B. Bobin, C. J. Bordé, J. Bordé, C. Breant, J. Mol. Spectrosc. 121 (1987) 91.

[17] I. Ozier, P. N. Yi, N. F. Ramsey, J. Chem Phys. 66 (1977) 143.

[18] B. C. John, P. von R. Schleyer, J. S. Binkley, A. P. John, J. Chem Phys. 64 (1976) 5142.

[19] J. T. H. Dunning, J. Chem Phys. 53 (1970) 2823.

[20] N. Godbout, D. R. Salahub, J. Andzelm, E. Wimmer, Can. J. Chem. 70 (1992) 560 .

[21] W. J. Hehre, R. Ditchfield, J. A. Pople, J. Chem Phys. 56 (1972) 2257. 
[22] K. Ruud, T. Helgaker, J. Olsen, P. Jorgensen, K. L. Bak, Chem. Phys. Lett. 235 (1995) 47.

[23] W. J. Hehre, L. Random, P. V. R. Schleyer, Ab Initio Molecular Orbital Theory, Wiley, new York, 1986.

[24] L. M. Crapo, G. W. Flynn, J. Chem Phys. 43 (1965) 1443.

[25] H. L. Tigelaar, W. H. Flygare, Chem. Phys. Lett. 7 (1970) 254.

[26] C. Chardonnet, Ph.D. thesis, Université Paris XIII (1989).

[27] C. W. Kern, N. L. William, J. Chem Phys. 37 (1962) 260.

[28] S. Mohanty, J. Chem Phys. 59 (1973) 4415.

[29] I. C. Sunney, T. P. Das, J. Chem Phys. 37 (1962) 1527.

[30] M. R. Baker, C. H. Anderson, N. F. Ramsey, Phys. Rev. 133 (1964) A1533. 
Table 1

Diamagnetic and paramagnetic electronic contributions to the scalar and tensorial spin-rotation constants $(\mathrm{KHz})$. The subscript "T" means that the correction due to the Thomas precession was taken into account.

$$
\begin{array}{lllllll}
\text { S and F basis sets } & c_{\mathrm{a}}^{\mathrm{ed}} & c_{\mathrm{a}, \mathrm{T}}^{\mathrm{ed}} & c_{\mathrm{d}}^{\mathrm{ed}} & c_{\mathrm{d}, \mathrm{T}}^{\mathrm{ed}} & c_{\mathrm{a}}^{\mathrm{ep}} & c_{\mathrm{d}}^{\mathrm{ep}} \\
\text { STO-3G, 6-31++G** } & -2.334 & -2.103 & -2.791 & -2.514 & -5.9006 & -4-481 \\
\text { STO-3G, DZP+Diffuse } & -1.611 & -1.452 & -1.831 & -1.650 & -6.6301 & -5.353 \\
\text { DZP, DZP+Diffuse } & -2.224 & -2.004 & -2.663 & -2.399 & -6.2086 & -4.920 \\
\text { TZVP (DFT), TZVP (DFT) } & -2.117 & -1.907 & -2.332 & -2.101 & -6.5207 & -5.382
\end{array}
$$

Table 2

Scalar $\left(c_{\mathrm{a}, \mathrm{T}}\right)$ and tensorial $\left(c_{\mathrm{d}, \mathrm{T}}\right)$ spin-rotation constants with Thomas precession correction (in $\mathrm{KHz}$ ) calculated in this work with with several basis sets.

$$
\begin{array}{lll}
\text { S and F basis sets } & c_{\mathrm{a}, \mathrm{T}} & c_{\mathrm{d}, \mathrm{T}} \\
\text { STO-3G, 6-31++G** } & -3.953 & -3.617 \\
\text { STO-3G, DZP+Diffuse } & -4.031 & -3.614 \\
\text { DZP, DZP+Diffuse } & -4.161 & -3.931 \\
\text { TZVP (DFT), TZVP (DFT) } & -4.377 & -4.095
\end{array}
$$

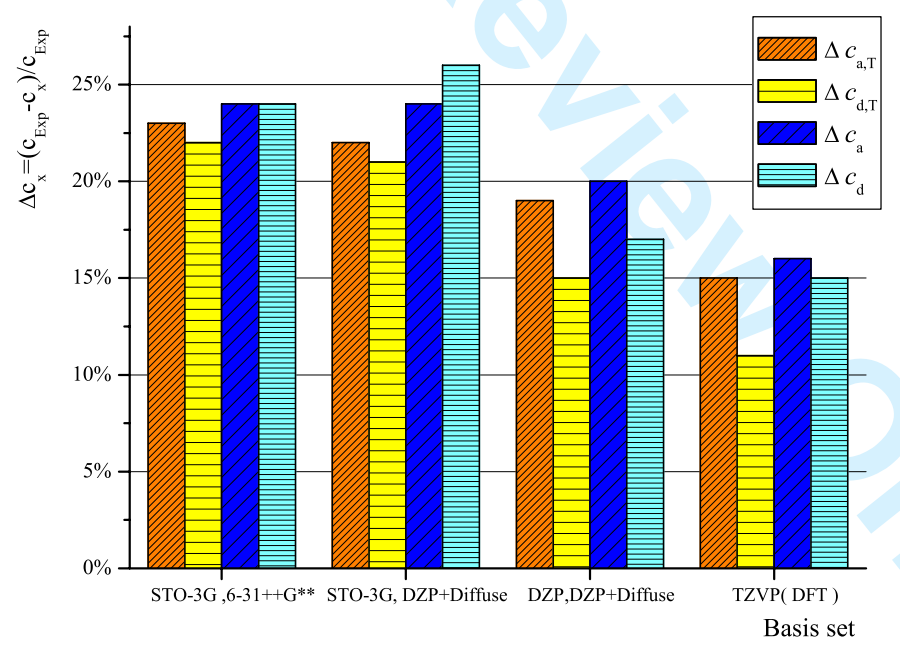

Fig. 1. Relative difference, for the scalar and tensorial spin-rotation constants, between the experimental values obtained by Chardonnet and Bordé [3,26] and the results obtained in this work, with $\left(\Delta c_{\mathrm{a}, \mathrm{T}}, \Delta c_{\mathrm{d}, \mathrm{T}}\right)$ and without $\left(\Delta c_{\mathrm{a}}, \Delta c_{\mathrm{d}}\right)$ the Thomas precession correction. 


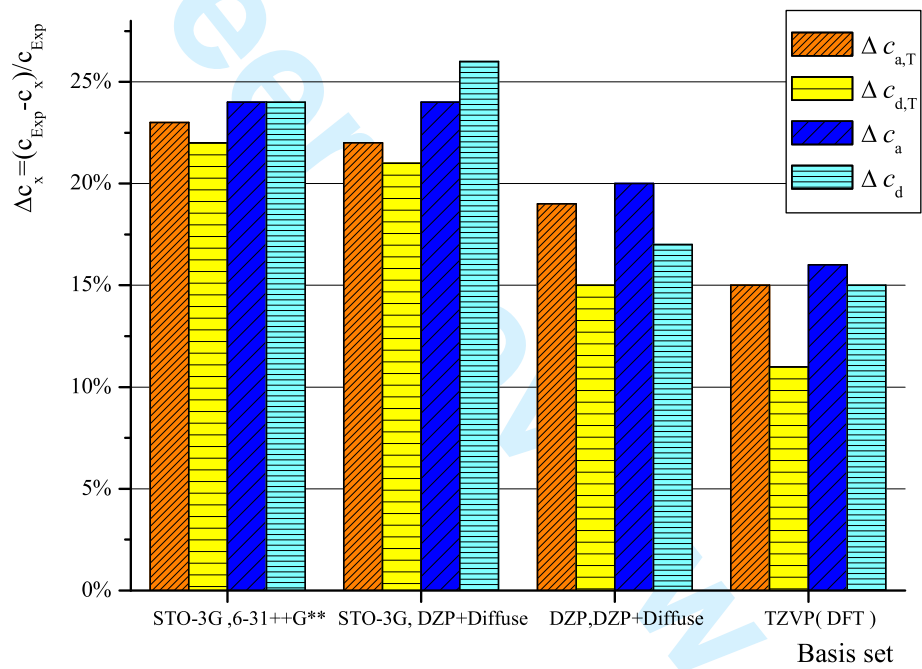

40

41

42

44

45

46

47

48

50

51

52

53

54

55

56 